\title{
Vermehrung und DNS-Synthese des Herpes-simplex-Virus nad Mitomycin C - Behandlung
}

\author{
Klaus Munk und Gerhard Sauer
}

Institut für Virusforschung Heidelberg

(Z. Naturforschg. 20 b, 671-677 [1965] ; eingegangen am 23. Februar 1965)

\begin{abstract}
In Herpes-Virus-infizierten HeLa-Kulturen findet sich während der ersten 6 Stdn. der Infektion, gemessen an ${ }^{3} \mathrm{H}$-Thymidineinbau, der gleiche Anteil an Zellen, die DNS synthetisieren (S-Phase), wie in nicht infizierten Kontrollkulturen. Nach 6 Stdn. p.i. steigt dieser Prozentsatz in infizierten Kulturen sehr rasch an. Durch Zugabe von Mitomycin C zu nicht infizierten Kulturen wird die DNS-Synthese fast ganz unterbunden. Das gleiche gilt auch für die infizierten Zellen, wenn Mitomycin zwischen 0 und $6 \mathrm{Stdn}$. p.i. angewendet wird. Wenn die Mitomycinbehandlung später als 6 Stdn. p.i. einsetzt, läßt sich ein hemmender Einfluß auf die DNS-Synthese nicht mehr nachweisen. Aus diesen Ergebnissen wird geschlossen, daß die Virus-DNS-Synthese 6 Stdn. p.i. beginnt und von nun an unabhängig von einer intakten zellulären DNS abläuft. Die Bildung von infektiösem Virus wird jedoch verhindert, wenn die Kulturen zwischen 0 und 12 Stdn. p.i. mit Mitomycin behandelt werden.
\end{abstract}

In der Wirtszelle induziert Herpes-simplex-Virus $(\mathrm{HsV})$ Prozesse, die direkt oder indirekt der Produktion von neuem Virus dienen und steuert die Reihenfolge und den zeitlichen Einsatz dieser Vorgänge. Gleichzeitig werden zelleigene Stoffwechselvorgänge gestört und die Struktur von Zelle und Kern verändert.

Durch frühere autoradiographische Untersuchungen ließ sich die Bildung des $\mathrm{HsV}$-induzierten DNSStoffwechsels im Kerninnenbereich der HeLa-Zelle beobachten ${ }^{1}$. Weitere Versuche an Mitomycin Cbehandelten, $\mathrm{HsV}$-infizierten Zellen zeigten, daß bestimmte, virusbedingte Vorgänge in den Zellen - wie die Syncytienbildung - auch dann ablaufen können, wenn die Virus-DNS-Synthese durch den Inhibitor gehemmt ist ${ }^{2}$.

Die Beziehungen zwischen Zell- und Virus-DNSStoffwechsel sind Gegenstand der vorliegenden Untersuchungen. Als Inhibitor der DNS-Synthese diente auch hier Mitomycin C.

\section{Material und Methoden}

\section{Virus}

Alle Versuche führten wir mit dem Herpes-VirusStamm „HOF“ durch. Die Eigenschaften dieses Stammes, der in Gewebekulturen großflächige Syncytien und im Dulbecco-Test große Plaques erzeugt, wurden früher beschrieben ${ }^{3}$. Die Viruspassagen erfolgten in HeLa-Zellkulturen.

\footnotetext{
1 K. Munk u. G. SaUer, Z. Naturforschg. 18 b, 211 [1963].

2 K. Munk u. G. Sauer, Virology 22, 153 [1964].
}

\section{Gewebekulturen}

Für die Gewebekulturen wurde ein HeLa-Dauerzellstamm verwendet. Als Nährmedium diente $\mathrm{H}$ a nk s sche Lösung mit 0,5\% Lactalbumin-Hydrolysat, 5\% Kälberserum und Antibiotica. Für einen Teil der Versuche wurden die HeLa-Zellen in der früher beschriebenen Weise ${ }^{1}$ auf Deckgläschen $2 \cdot 30 \mathrm{~mm}$ angezüchtet und in Kulturröhrchen unter $5,0 \mathrm{ml}$ Nährmedium gehalten. In anderen Fällen verwendeten wir HeLa-Zellkulturen in $100 \mathrm{ml}$-Erlenmeyer-Kölbchen. Die Versuche begannen 72 Stdn. nach dem Anlegen der Kulturen, wenn sich ein geschlossener Zellrasen gebildet hatte. Jedes Kölbchen enthielt etwa $7 \cdot 10^{6}$ Zellen. Die Beimpfung erfolgte so, daß sich eine Multiplicity von 1 ergab.

\section{Virustitration}

Die Virustitration erfolgte nach der Methode von Dulbecco und Vogt ${ }^{4}$, deren Anpassung an das $\mathrm{HsV}$ HeLa-Zell-System früher beschrieben worden war ${ }^{3}$. Wegen der nur geringen Virustiter, die in den kleinen Deckglas-Gewebe-Kulturen und auch in den KölbchenKulturen zu erreichen waren, mußten wir die Verdünnungsstufen in Zweierpotenzen anlegen.

\section{Radionuklide}

Das ${ }^{3}$ H-Thymidin (spez. Aktivität $2,4 \mathrm{C} / \mathrm{mMol}$ ) wurde vom Radiochemical Centre in Amersham Buckinghamshire bezogen.

\section{Autoradiographische Methode}

Nach Fixierung der Präparate in Alkohol-Eisessig $(6: 1)$ und sofortiger Überführung in 80-proz. Alkohol wurden die Deckglas-Gewebekulturen luftgetrocknet

${ }^{3}$ K. Munk u. D. Donner, Arch. ges. Virusforsch. 13, 529 [1963].

4 R. Dulbecco u. M. Vogt, J. exp. Medicine 99, 167 [1954]. 
und mit der Zellschicht nach oben mittels Eukitt auf Objektträger aufgeklebt. Die gut getrockneten Präparate wurden eine Stde. fließend gewässert und daraufhin mit einem Gelatineüberzug versehen, um ein besseres Haften des stripping-Films zu ermöglichen (5 g Fotogelatine und $0,5 \mathrm{~g}$ Chromalaun auf $1000 \mathrm{ml}$ dest. Wasser). Während des Aufbringens des KodakAR-10-Films stellten wir eine rel. Luftfeuchtigkeit von $45 \%$ mittels eines Hygrostaten ein. Die Expositionszeit betrug drei Tage. Die Präparate wurden mit Amidol entwickelt und mit Ehrlich schem Hämatoxylin gefärbt.

\section{Mitomycin $C$}

Das Mitomycin C stammt von der Firma KyowaHakko-Kogyo-Co. Ltd. Tokio.

\section{Versuche und Ergebnisse}

Vermehrungsdauer des Hs V-Stammes "H O F“

Als Grundlage für die Beurteilung der Ergebnisse mußten zuerst der Vermehrungsablauf und das zeitliche Auftreten der Zell- bzw. Kern-Veränderungen in HeLa-Zellkulturen nach Infektion mit dem hier verwendeten Virusstamm „HOF“ untersucht werden.

Zur Bestimmung des Virustiters wurden $72 \mathrm{Stdn}$. alte HeLa-Zellkulturen in 100-ml-E r l e n m e y e r Kölbchen infiziert. 3 Stdn. p.i. wurde das Medium gewechselt und die Kulturen zweimal mit gepufferter NaCl-Lösung gewaschen. Der Gehalt an freiem Virus im Nährmedium und an zellgebundenem Virus, das

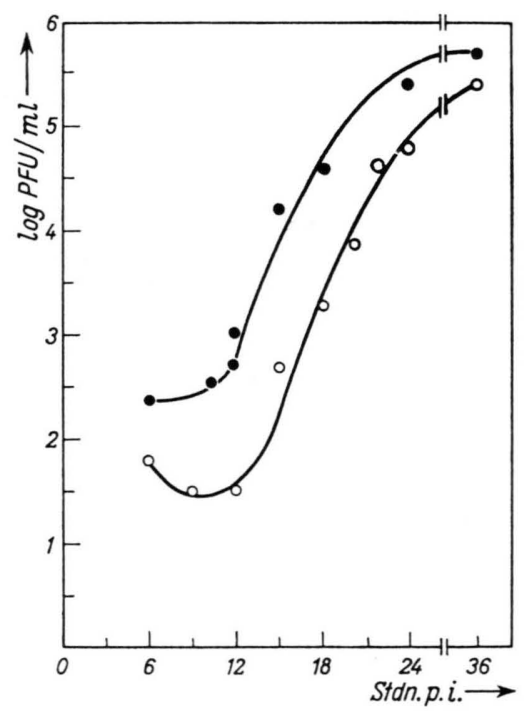

Abb. 1. Wachstumskurve von Herpes-simplex-Virus, Stamm Hofmeister. $-\bullet=$ Zellgebundenes Virus, $\mathrm{-}-\mathrm{O}=$ Freies Virus, Mittelwerte aus drei Titrationsversuchen. aus den Zellen nach dreimaligem Einfrieren und Auftauen freigemacht worden war, wurde gesondert bestimmt.

Zwischen 10 und 12 Stdn. p.i. steigt der Titer des zellgebundenen Virus an (Abb. 1). Die Freisetzung der Viruspartikel erfolgt kurze Zeit später. Die Zellbzw. Kernveränderungen sind quantitativ nur schwer zu bestimmen. Etwa 8 Stdn. p. i. läßt sich die Entstehung des Randchromatins beobachten. Die Syncytien entstehen zwischen der 10. und 12. Stde. p.i. In infizierten Zellkulturen sind zu diesen Zeiten nach der Infektion die Zellen annähernd 100. proz. cytopathisch verändert. Soweit es die Methode zuläßt, ist daraus zu schließen, daß der Vermehrungsablauf in einem Streuungsbereich von wenigen Stdn. annähernd synchron verläuft.

$$
\begin{gathered}
\text { DNS-Stoffwechsel in der infizierten } \\
\text { Zelle }
\end{gathered}
$$

Der DNS-Stoffwechsel in der $\mathrm{HsV}$-infizierten Zelle sollte hier im Ablauf der Vermehrung verfolgt und quantitativ an Hand von Autoradiographien ausgewertet werden.

Die auf den Deckgläschen angelegten HeLa-Zellkulturen wurden mit $\mathrm{HsV}$ infiziert und erhielten zu bestimmten Zeiten p.i. einen einstündigen Puls mit $0,25 \mu \mathrm{C}{ }^{3} \mathrm{H}$-Thymidin $/ \mathrm{ml}$. Nach diesem Thymidinpuls wurden die Kulturen sofort entnommen, fixiert und entsprechend der autoradiographischen Technik behandelt. Zur Beurteilung der Einbaurate wurde die Menge des aufgenommenen Thymidins in Form der Silberkörner pro Zellkern herangezogen. Die Werte für jedes Präparat wurden durch zweimaliges, unabhängiges Zählen von je 30 markierten Kernen ermittelt.

Die Ergebnisse dieser dreifach wiederholten Versuche sind an einem Beispiel in der Abb. 2 dargestellt. Sie zeigen das Ausmaß der ${ }^{3} \mathrm{H}$-Thymidin-Aufnahme in infizierten Zellen bei einem 1-stdg. Puls zu bestimmten Zeiten p.i. Zur Kontrolle und zur Bestimmung der DNS-Synthese-Kapazität, gemessen an der Thymidin-Aufnahme bei nicht infizierten Zellen, erhielten gleichaltrige HeLa-Zellkulturen am Beginn und am Ende des Versuches den quantitativ gleichen 1-stdg. ${ }^{3} \mathrm{H}$-Thymidinpuls. Wie die Abb. 2 zeigt, inkorporieren Kontrollkulturen etwa 60 grains/Kern.

In infizierten Zellen sinkt der Einbau von Thymidin während der ersten Stde. p.i. sehr stark ab (ausgezogene Kurve). Darin stimmen alle Versuche zeitlich genau überein. Nach diesem Abfall steigt die DNS-Synthese zwischen der 4. und 8. Stde. wieder auf das Anfangsniveau an. Nach dieser Zeit wird die Einbaurate in der infizierten Zelle fortlaufend geringer. 


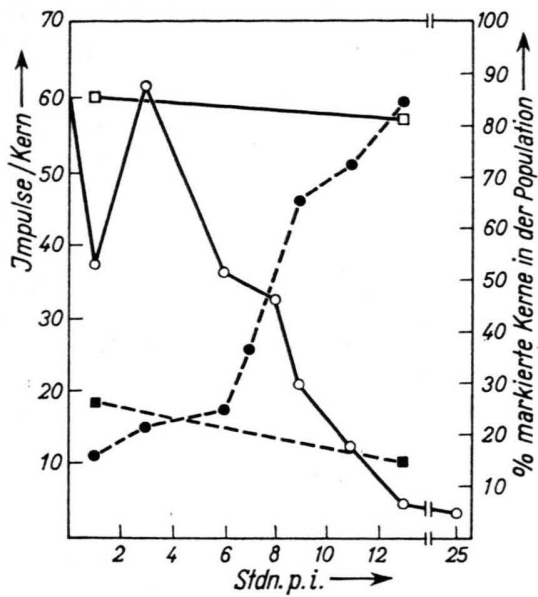

Abb. 2. ${ }^{3} \mathrm{H}$-Thymidin-Einbaurate in die DNS nicht infizierter und Herpes-Virus-infizierter HeLa-Zellen. Zu verschiedenen Zeitpunkten wurden infizierte und nicht infizierte HeLa-Deckglaskulturen mit $0,25 \mu \mathrm{C}{ }^{3} \mathrm{H}$-Thymidin/ml I Stde. lang inkubiert, anschließend sofort fixiert und die Präparate autoradiographisch ausgewertet. Die durch Auszählung ermittelten Silberkörner/Kern sind jeweils am Ende der Pulszeit eingetragen. $\square-\square=$ Nicht infiziert, $\circ-0=$ Infiziert. Der sich unter den gleichen experimentellen Bedingungen ergebende prozentuale Anteil markierter Zellkerne an der Gesamtpopulation ist ebenfalls in das Diagramm eingetragen. $\mathbf{-}-\mathbf{-}=$ Nicht infiziert, $\bullet-\bullet=$ Infiziert.

Die autoradiographische Methodik erlaubt an den gleichen Präparaten noch eine weitere, sehr aufschlußreiche Auswertung. Mit ihrer Hilfe läßt sich der Prozentsatz der Zellen in der Gesamtpopulation bestimmen, die während des 1-stdg. Pulses ${ }^{3} \mathrm{H}$-Thymidin aufgenommen haben. Diese Resultate sind ebenfalls in Abb. 2 wiedergegeben (gestrichelte Kurve, ausgefüllte Kreise). Sie sind Mittelwerte, gewonnen durch zweimaliges unabhängiges Auszählen von je 6 Blickfeldern bei 700-facher Vergrößerung. In den nicht infizierten Kontrollkulturen sind $15-30 \%$ der Zellen markiert (Abb. 2). Da die normale Zelle angebotenes Thymidin nur während der S-Phase des Mitosecyclus aufnimmt, während der die Zelle ihre DNS synthetisiert, repräsentiert dieser Prozentsatz solche Zellen, die sich während des 1-stdg. ${ }^{3} \mathrm{H}$-Thymidinpulses in irgendeinem Stadium der S-Phase befunden haben.

Auch in den infizierten Zellkulturen nehmen während der ersten $6-7$ Stdn. p.i. $20-30 \%$ der Zellen ${ }^{3} \mathrm{H}$-Thymidin auf (Abb. 2). Nach der 6. bis 7. Stde. p.i. steigt der Prozentsatz markierter Zellen in infizierten Kulturen sehr stark an, um bald einen Wert von etwa $80 \%$ zu erreichen.
Virusvermehrung und DNS-Stoffwechselunter Mitomycin C.

Einwirkung

Frühere Untersuchungen ${ }^{2}$, die sich mit den Beziehungen zwischen DNS-Stoffwechsel und Virusvermehrung bzw. cytopathischen Veränderungen der Wirtszelle unter Einwirkung von Mitomycin C (MC) auf die Wirtszelle befaßten, sollten hier mit der Fragestellung fortgeführt werden, wieweit die Prozesse während der Virusvermehrung von einer intakten Funktion der Zell-DNS abhängig sind.

a) Prüfung der MC-Konzentration und -Einwirkungsdauer auf die Virus-Neubildung und den DNS-Stoffwechsel der infizierten und nicht in fizierten Zelle

In Vorversuchen wurde die Wirkung der MCKonzentration und der Einfluß der Einwirkungsdauer auf den DNS-Stoffwechsel der infizierten und nicht infizierten Zelle sowie auf die Virusproduktion geprüft.

Zur Bestimmung der Virusvermehrung wurden HeLa-Zellen in E rlenmeye r-Kölbchen mit HsV beimpft. Zugleich erhielten die Kulturen jeweils 8 bzw. $16 \mu \mathrm{g} / \mathrm{ml}$ MC. $\mathrm{Zu}$ bestimmten Zeiten p.i. wurde das MC-haltige Medium entfernt, die Kulturen mehrmals

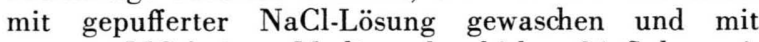
neuem, MC-freiem Medium beschickt. 24 Stdn. p.i. wurde das Kulturmedium entnommen und der Virustiter im Plaquetest bestimmt.

Die Virusneubildung hängt von der Konzentration und Einwirkungsdauer des Mitomycins ab (Tab. 1).

\begin{tabular}{|c|c|c|}
\hline $\begin{array}{c}\text { Mitomycin C } \\
\text { Menge } \\
{[\mu \mathrm{g}]}\end{array}$ & $\begin{array}{c}\text { Mitomycin C } \\
\text { Einwirkungs- } \\
\text { dauer [Stdn.] }\end{array}$ & $\begin{array}{c}\text { Virustiter } \\
\text { in \% der } \\
\text { Kontrolle }\end{array}$ \\
\hline & 1 & 3,12 \\
& 2 & 3,12 \\
& 3 & 0,19 \\
& 4 & 0,05 \\
& 5 & 0,05 \\
\hline 16 & 1 & 3,12 \\
& 3 & 0,76 \\
& 4 & 0,05 \\
\hline
\end{tabular}

Tab. 1. Virusausbeute in Abhängigkeit von der Mitomycin CDosierung und -Einwirkungszeit. HeLa-Deckglaskulturen wurden zugleich mit der Infektion mit $8 \mu \mathrm{g}$ Mitomycin $\mathrm{C} / \mathrm{ml}$ oder $16 \mu \mathrm{g}$ Mitomycin $\mathrm{C} / \mathrm{ml} \mathrm{1,2,3,4}$ und $5 \mathrm{Stdn}$. lang gemeinsam inkubiert. Nach den verschiedenen Einwirkungszeiten wurde das Mitomycin durch Waschen mit BSS entfernt und die Blättchen in neues Kulturmedium überführt. Die Titration des im Medium befindlichen Virus geschah 24 Stdn. nach Impfung. Die erzielten Ausbeuten sind in Prozent der Kontrolle aufgetragen. Als Kontrolle diente eine infizierte und nicht mit Mitomycin behandelte Deckglaskultur, bei welcher das freigesetzte Virus 24 Stdn. p. i. titriert wurde. 
Schon nach einer 2-stdg. Einwirkung von $16 \mu \mathrm{g} / \mathrm{ml}$ MC läßt sich die Ausbeute an infektiösem Virus fast ganz unterdrücken. Diese am HsV-HeLa-Zell-System gewonnenen Ergebnisse bestätigen die früher von Reich und Franklin ${ }^{5}$ veröffentlichten Befunde.

Die MC-Wirkung auf die DNS-Synthese der infizierten und nicht infizierten Zelle untersuchten wir autoradiographisch durch Bestimmung der ${ }^{3} \mathrm{H}$-Thymidin-Aufnahme.

Die Deckglas-Kulturen wurden zum Zeitpunkt der Infektion in Nährmedium gebracht, das $16 \mu \mathrm{g} / \mathrm{ml} \mathrm{MC}$ enthielt. Am Ende der gewünschten MC-Einwirkungszeit wurden sie gewaschen und für eine weitere Stde. in Nährmedium mit $0,5 \mu \mathrm{C} / \mathrm{ml}{ }^{3} \mathrm{H}$-Thymidin überführt. Nach dem Thymidinpuls und nach nochmaligem Waschen in gewärmter $\mathrm{H}$ a n k s scher Lösung wurden die Kulturen bis 24 Stdn. p.i. weiter inkubiert, um die gleichen Bedingungen wie bei den Titrationsversuchen einzuhalten. Anschließend wurden sie fixiert und autoradiographisch ausgewertet. Die nicht infizierten Kontrollkulturen wurden in der gleichen Weise behandelt.

In nicht infizierten Zellen wird die ${ }^{3} \mathrm{H}$-ThymidinAufnahme mit zunehmender MC-Einwirkungsdauer zunehmend gehemmt (Tab.2). Die Werte der ${ }^{3} \mathrm{H}$ Thymidin-Aufnahme in den infizierten Zellen zeigten ein bemerkenswertes Ergebnis (Tab. 2). Auch hier besteht eine Beziehung zwischen zunehmender

\begin{tabular}{|c|c|c|c|c|}
\hline \multirow[b]{2}{*}{$\begin{array}{l}16 \mu \mathrm{g} \\
\text { Mito- } \\
\text { mycin C } \\
\text { Einwir- } \\
\text { kungs- } \\
\text { dauer } \\
\text { [Stdn.] }\end{array}$} & \multicolumn{2}{|c|}{ nicht infizierte Zellen } & \multicolumn{2}{|c|}{ infizierte Zellen } \\
\hline & $\begin{array}{c}{ }^{3} \mathrm{H}- \\
\text { Thymidin } \\
\text { Einbau } \\
\text { [counts/ } \\
\text { nucl.] }\end{array}$ & $\begin{array}{c}\text { markierte } \\
\text { Zellen der } \\
\text { Kultur } \\
{[\%]}\end{array}$ & $\begin{array}{c}{ }^{3} \mathrm{H}- \\
\text { Thymidin } \\
\text { Einbau } \\
\text { [counts/ } \\
\text { nucl. }]\end{array}$ & $\begin{array}{c}\text { markierte } \\
\text { Zellen der } \\
\text { Kultur } \\
{[\%]}\end{array}$ \\
\hline $\begin{array}{l}1 \\
2 \\
3 \\
4 \\
5\end{array}$ & $\begin{array}{r}27,5 \\
26,5 \\
17,5 \\
10,5 \\
6,5\end{array}$ & $\begin{array}{r}38,4 \\
19,0 \\
12,0 \\
11,5 \\
5,4\end{array}$ & $\begin{array}{r}20,5 \\
13,0 \\
10,5 \\
8,5 \\
5,5\end{array}$ & $\begin{array}{r}33 \\
15,6 \\
11,4 \\
9,6 \\
7,4\end{array}$ \\
\hline
\end{tabular}

Tab. 2. ${ }^{3} \mathrm{H}$-Thymidin-Einbaurate in die DNS infizierter und nicht infizierter Zellkerne nach unterschiedlicher Mitomycin CDosierung und -Einwirkungszeit. Infizierte und nicht infizierte HeLa-Deckglaskulturen wurden wie bei Tab. 1 beschrieben mit Mitomycin C verschieden lang inkubiert. Nach Beendigung der Mitomycin-Inkubationszeit wurden die Zellen gewaschen und für $5 \mathrm{Stdn}$. in Medium überführt, welches $0,5 \mu \mathrm{C}$ ${ }^{3} \mathrm{H}$-Thymidin pro ml enthielt. Nachdem sie daraufhin ein weiteres Mal gewaschen worden waren, kamen sie in Medium mit dem 100 -fachen Überschuß an nicht radioaktivem ${ }^{3} \mathrm{H}$-Thymidin. Die Fixierung und autoradiographische Auswertung geschah 24 Stdn. nach Impfung. In der Tabelle sind die Silberkörner pro Zellkern und in getrennten Spalten der Prozentsatz markierter Zellkerne in den Kulturen enthalten.

5 E. Reich u. R. M. Franklin, Proc. nat. Acad. Sci. [Wash.] 47,1212 [1961].
MC-Einwirkungszeit und verminderter ${ }^{3} \mathrm{H}$-ThymidinAufnahme. Verglichen mit den Aufnahmewerten der nicht infizierten Zellen fanden wir in den infizierten, MC-behandelten Zellen eine stärkere Hemmung des ${ }^{3} \mathrm{H}$-Thymidin-Einbaues. Nach diesen Ergebnissen ist die Hemmung der ${ }^{3} \mathrm{H}$-Thymidin-Aufnahme in Mitomycin-behandelten, infizierten Zellen stärker als in Mitomycin-behandelten, nicht infizierten Zellen. Sie ist in gleicher Weise wie in den nicht MC-behandelten, infizierten Kulturen am stärksten zwischen 2 und 3 Stdn. p.i. ausgeprägt (vgl. Abb. 2, ausgezogene Kurve).

b) Virusvermehrung und Virus-DNS-Synthese in Abhängigkeit von der Zell-DNS, geprüft nach MCEinwirkung zu verschiedenen Zeiten nach der Infektion

Wenn die MC-Einwirkung vor oder zugleich mit der Infektion stattfand, konnten REICH und FrankLIN $^{5}$ eine Vermehrung des Vaccine-Virus gänzlich unterdrücken. Für unsere bisherigen Versuche, bei denen MC zugleich mit der Infektion gegeben wurde, gilt das gleiche. Darüber hinaus sollte hier untersucht werden, wie sich die Virusvermehrung und Virus-DNS-Synthese verhalten, wenn zu verschiedenen Zeiten nach der Infektion die MC-Einwirkung beginnt.

Die Prüfung der Virusvermehrung geschah hier in gleicher Weise wie oben beschrieben an HeLa-Zellkulturen in Erlenmeyer-Kölbchen. Ein Teil dieser Kulturen erhielt vor der Infektion 5 Stdn. lang $20 \mu \mathrm{g}$ bzw. $40 \mu \mathrm{g} \mathrm{MC} / \mathrm{ml}$. Dann wurde das MC-haltige Medium entfernt, die Kulturen 2-mal mit PBS gewaschen und mit Virus beimpft. 3 Stdn. p.i. wurde das nicht adsorbierte Virus entfernt, die Kulturen erneut 2-mal gewaschen und nach 26 Stdn. p.i. das Virus titriert. Hierbei betrug die Virusmenge nur 1,55\% der nicht behandelten Kontrolle.

Andere Kulturen erhielten 5 Stdn. lang Mitomycin zu verschiedenen Zeiten nach der Infektion. Nach Beendigung der MC-Einwirkung wurden die Kulturen 2-mal gewaschen und für weitere $10-15$ Stdn. unter MCfreiem Medium gehalten; das während dieser Zeit freigesetzte Virus wurde dann titriert. Die Ergebnisse sind in Tab. 3 als $\%$ der Kontrollen aus nicht behandelten Zellen mit gleicher Vermehrungsdauer eingetragen.

Die Virusausbeute ist sowohl in MC-vorbehandelten Zellen als auch in solchen, die während der ersten 5 Stdn. p.i. MC erhielten, stark herabgesetzt. Mitomycindosen von $20 \mu \mathrm{g} / \mathrm{ml}$, welche zu späteren Zeitpunkten nach der Infektion gegeben werden, erlauben nur eine geringe Virusausbeute. Mit $40 \mu \mathrm{g}$ Mitomycin/ml läßt sich bis 12 Stdn. nach der Infek- 
tion jegliche Virusvermehrung unterbinden. Erst von $13 \mathrm{Stdn}$. nach der Infektion an erreicht die Virusausbeute auch unter der hohen Mitomycindosierung von $40 \mu \mathrm{g} / \mathrm{ml}$ stets den Wert, der in Kontrollkulturen zur gleichen Zeit enthalten war. Dieser Zeitpunkt deckt sich etwa mit dem ersten Auftreten des zellgebundenen Virus (Abb. 1). Daraus schließen wir, daß einmal fertiggestelltes Virus durch Mitomycin nicht mehr angegriffen werden kann. Dagegen kommt eine weitere Bildung infektiöser Virusteilchen nicht mehr zustande.

Um der Frage nachzugehen, wie sich die DNSSynthese unter den gleichen experimentellen Bedingungen verhält, wurden Versuche mit Deckglaskulturen angestellt.

Sie wurden in gleicher Weise wie die oben beschriebenen Virustitrations-Versuche angesetzt, nur mit dem Unterschied, daß sofort nach der 5-stdg. Einwirkung von $20 \mathrm{bzw}$. $40 \mu \mathrm{g} \mathrm{MC} / \mathrm{ml}$ ein einstündiger ${ }^{3} \mathrm{H}$-Thymidinpuls von $0,5 \mu \mathrm{C} / \mathrm{ml}$ gegeben wurde. Nach Beendigung des Pulses wurden die Kulturen fixiert und autoradiographisch ausgewertet. Als Kontrollen dienten nicht infizierte, MC-behandelte Zellkulturen.

Die durch grain counting ermittelten Ergebnisse sind in Tab. 3 in Prozenten der Kontrollen eingetragen. In nicht infizierten, mit $40 \mu \mathrm{g} / \mathrm{ml}$ MC-behandelten HeLa-Zellen beträgt der ${ }^{3} \mathrm{H}$-Thymidin-Einbau stets $10 \%$ der nicht behandelten Kontrollkulturen. Ganz anders verhält sich dagegen die ${ }^{3} \mathrm{H}$-ThymidinEinbaurate in MC-behandelten, infizierten Kulturen. Nur bei einer MC-Einwirkung von $40 \mu \mathrm{g} / \mathrm{ml}$ zugleich mit der Infektion liegen die Einbauwerte in dem gleichen Bereich wie bei MC-behandelten Kontrollkulturen (Tab. 3). Beginnt die MC-Behandlung 3 Stdn. p.i., dann zeigt sich bereits eine Zunahme der DNS-Synthese. Wird MC 6 Stdn. p.i. gegeben, so ist die Einbaurate von ${ }^{3} \mathrm{H}$-Thymidin ebenso hoch wie bei unbehandelten, infizierten Zellen. Spätere MC-Gaben zeigen ebensowenig einen Einfluß auf die Synthese der virusinduzierten DNS. Diese Befunde zeigen, daß durch MC die celluläre DNS-Synthese blockiert werden kann. Dagegen kann die Virus-induzierte DNS-Synthese nach 6 Stdn. p.i. nicht mehr gehemmt werden, wenn die Vorgänge während der ersten Stdn. nach der Infektion ungestört ablaufen konnten. Es ist somit möglich, die Zell- und die Virus-induzierte DNS-Synthese nach einer bestimmten Phase der Infektion voneinander zu trennen.

${ }^{6}$ A. S. Kaplan u. T. Ben-Porat, Virology 19, 205 [1963].

\begin{tabular}{|c|c|c|c|c|}
\hline \multirow{2}{*}{$\begin{array}{l}\text { Beginn einer } \\
5 \text { stündigen } \\
\text { Mitomycin C } \\
\text { Einwirkung } \\
\text { [Stdn./p.i.] }\end{array}$} & \multicolumn{2}{|c|}{$\begin{array}{c}\text { Virustiter } \\
\text { in \% der } \\
\text { unbehandelten } \\
\text { infizierten Kontrolle }\end{array}$} & \multicolumn{2}{|c|}{$\begin{array}{c}{ }^{3} \mathrm{H}-\text { Thymidin } \\
\text { Einbau in \% der } \\
\text { unbehandelten } \\
\text { infizierten Kontrolle }\end{array}$} \\
\hline & $20 \mu \mathrm{g} / \mathrm{ml}$ & $40 \mu \mathrm{g} / \mathrm{ml}$ & $20 \mu \mathrm{g} / \mathrm{ml}$ & $40 \mu \mathrm{g} / \mathrm{ml}$ \\
\hline $\begin{array}{r}0 \\
3 \\
6 \\
9 \\
12\end{array}$ & $\begin{array}{r}3,03 \\
2,2 \\
9,5 \\
7,3 \\
13,5\end{array}$ & $\begin{array}{l}0,39 \\
0,01\end{array}$ & $\begin{array}{l}18 \\
43 \\
98,5 \\
100 \\
100\end{array}$ & $\begin{array}{r}9,5 \\
39,2 \\
84 \\
97,5\end{array}$ \\
\hline
\end{tabular}

Tab. 3. Abhängigkeit der Virusausbeute und der ${ }^{3} \mathrm{H}-\mathrm{Thymi}-$ din-Einbaurate vom Beginn der Mitomycineinwirkung. HeLaMonolayerkulturen in $100 \mathrm{ml} \mathrm{Erle} \mathrm{n} \mathrm{m} \mathrm{e} \mathrm{ye} \mathrm{r}$-Kölbchen wurden 3 Tage nach dem Versenieren infiziert und zu verschiedenen Zeitpunkten nach der Infektion mit 20 bzw. $40 \mu \mathrm{g}$ Mitomycin C pro ml $5 \mathrm{Stdn}$. lang inkubiert und danach 2-mal mit BSS gewaschen. Sie wurden anschließend mit neuem Kulturmedium versehen und das freigesetzte Virus $26 \mathrm{Stdn}$. p.i. titriert. In der ersten Spalte der Tabelle ist der Beginn der verschiedenen Mitomycin-Einwirkungszeiten in Stdn. p.i. aufgetragen. Die zweite Spalte enthält die Titerangaben ausgedrückt in Prozent einer nicht mit Mitomycin behandelten Kultur, deren freigesetztes Virus ebenfalls 26 Stdn. p. i. titriert wurde. Die Daten sind Mittelwerte aus 5 Versuchen. Die ${ }^{3} \mathrm{H}$-Thymidin-Einbaurate in die DNS infizierter und in der gleichen Weise behandelter Deckglaskulturen ist in der rechten Spalte der Tabelle aufgeführt. Die infizierten Deckglaskulturen wurden nach den 5-stdg. Mitomycinpulsen gewaschen und für jeweils eine Stde. einem ${ }^{3} \mathrm{H}$-Thymidinpuls von $0,5 \mu \mathrm{C} / \mathrm{ml}$ ausgesetzt. Daraufhin wurden sie fixiert und autoradiographisch ausgewertet. Die Ergebnisse sind gleichfalls in Prozent einer unbehandelten infizierten Kontrolle (vgl. Abb. 2) ausgedrückt. Nicht infizierte und entsprechend mit Mitomycin behandelte HeLa-Kulturen inkorporierten unabhängig vom Beginn der Mitomycineinwirkung stets nur etwa $10 \%{ }^{3} \mathrm{H}$-Thymidin einer normalen HeLa-Kultur.

\section{Diskussion}

In einer früheren Arbeit ${ }^{1}$ haben wir autoradiographisch gezeigt, daß in $\mathrm{HsV}$-infizierten HeLa-Zellen nach den ersten Stdn. der Infektion der zelleigene DNS-Stoffwechsel erlischt. Kaplan und BENPorat kamen an Pseudorabies-Virus-infizierten Zellen $\mathrm{zu}$ dem gleichen Ergebnis ${ }^{6}$. Einige Stdn. nach der Infektion beginnt eine Virus-induzierte DNS. Synthese im Kerninnenbereich. In der vorliegenden Arbeit sollen diese Verhältnisse durch grain counting quantitativ untersucht werden. Wir fanden dabei unmittelbar nach der Infektion einen verringerten ${ }^{3} \mathrm{H}$-Thymidin-Einbau in die infizierten Kerne, der durch den vor Versuchsbeginn vorgenommenen Mediumwechsel bedingt sein kann (Abb. 2, ausgezogene Kurve). In den folgenden Stdn. p.i. erreicht die DNS-Synthese wieder die Ausgangswerte nicht infizierter Zellen und wird dann geringer. 
Diese aus den Einbauraten pro Kern erhaltenen Werte erlauben keinen Rückschluß auf die Eigenschaften der DNS. Ob es sich dabei um Zell- oder VirusDNS handelt, muß aus anderen Befunden ermittelt werden. Wenn wir den Anteil der markierten Zellen in infizierten sowie nicht infizierten Zellkulturen betrachten (Abb. 2, gestrichelte Kurven), so zeigt es sich, daß er in beiden Fällen bis 6 Stdn. p.i. mit $20-30 \%$ im gleichen Bereich liegt. Daraus schließen wir, daß es sich in diesem Zeitraum zunächst um einen zelleigenen DNS-Stoffwechsel handeln muß, welcher unter dem Einfluß der Infektion ausklingt. Auch Kaplan und Ben-Porat ${ }^{6}$ sowie Roizman und Roane $^{7}$ fanden bei Pseudorabies- bzw. HsV-infizierten Zellen ein ähnliches Verhalten.

6 Stdn. p.i. wird sehr rasch ein steigender Prozentsatz von Zellen in die Lage versetzt, Thymidin einzubauen (Abb. 2, gestrichelte Kurve). Diese plötzliche Überführung der Zellpopulation in eine neue Synthesephase deuten wir als den Beginn der VirusDNS-Synthese. Diese Folgerung wird durch die noch $\mathrm{zu}$ besprechenden, mit Mitomycin erhaltenen Versuchsergebnisse bestätigt. Inzwischen durchgeführte Zentrifugationen im $\mathrm{CsCl}_{2}$-Dichtegradienten führten zu den gleichen Ergebnissen. In dem Zeitraum zwischen 3 Stdn. und 6 Stdn. p.i. wird nur celluläre DNS synthetisiert, während sich in dem Intervall zwischen $7 \mathrm{Stdn}$. und $10 \mathrm{Stdn}$. p.i. neben überwiegender Virus-DNS noch eine ausklingende Zell-DNSSynthese nachweisen ließ. Diese Ergebnisse werden an anderer Stelle veröffentlicht. Auch bei $\mathrm{HsV}$-infizierten BHK-21-Zellen ${ }^{8}$ und HEP-2-Zellen ${ }^{7}$ fand sich auf Grund von Dichtegradienten-Zentrifugationen eine annähernde zeitliche Übereinstimmung dieses Vorganges.

Inwieweit die Virus-induzierte DNS-Synthese und die damit verbundene Vermehrung infektiöser Viruspartikel von der Funktion einer intakten Zell-DNS abhängen, wurde mit Hilfe von Mitomycin C untersucht. Wenn Mitomycin in hinreichend hoher Dosierung vor oder zugleich mit der Infektion verwendet wird, verhindert es die Bildung von DNS-Viren wie Vaccinia ${ }^{5}$.

Auch Ben-Porat, Reissig und Kaplan ${ }^{9}$ konnten durch Anwendung von Mitomycin C vor und zugleich mit der Infektion die Bildung von infektiösem Pseudorabies-Virus blockieren. Wenn sich die ver-

\footnotetext{
7 B. Roizman u. P. R. Roane, Virology 22, 262 [1964].

8 W. C. Russell, E. Gold, H. M. Keir, H. Omura, D. H. Watson u. P. Wildy, Virology 22, 103 [1964].
}

wendeten Kaninchen-Nieren-Kulturen allerdings in der stationären Phase des Wachstums befanden, lief - so vermuten die Autoren - als Folge einer massiven Induktion von Enzymsystemen eine VirusDNS-Synthese ab. Diese unter Mitomycin-Einfluß gebildete DNS war nicht infektiös.

In unseren Versuchen konnten wir durch Mitomycingaben vor oder zugleich mit der Infektion ebenfalls die Ausbeute an infektiösem Virus verhindern. Durch Modifizierung des experimentellen Ansatzes ergaben sich jedoch neue Befunde. Wenn eine genügend hohe Mitomycindosis von $40 \mu \mathrm{g} / \mathrm{ml}$ zu verschiedenen Zeitpunkten nach der Infektion - nämlich bis 12 Stdn. p.i. - angewendet wird, erscheint kein infektiöses Virus (Tab. 2). Geringere Mitomycin-Konzentrationen von $20 \mu \mathrm{g} / \mathrm{ml}$ erlauben zwar eine gewisse Virussynthese, zeigen aber prinzipiell ein ähnliches Verhalten wie höhere Konzentrationen. Sehr aufschlußreich ist die unter den oben genannten experimentellen Bedingungen ablaufende DNS. Synthese (Tab. 2). Wenn Mitomycin gemeinsam mit der Infektion den Kulturen zugefügt wird, bleibt die DNS-Synthese auf ein Minimum reduziert. Auf S. 674 wurde bereits auf die Tatsache hingewiesen, daß in Mitomycin-behandelten, infizierten Kulturen eine noch stärkere Hemmung der DNS-Synthese auftritt als in den als Kontrolle dienenden Mitomycinbehandelten, nicht infizierten Zellen. Die bei der normalen Virusvermehrung unmittelbar nach der Infektion einsetzende starke Hemmung der DNSSynthese (Abb. 2, ausgezogene Kurve) läßt sich demnach auch unter Mitomycin-Einfluß nachweisen.

Wenn zwischen dem Infektionszeitpunkt und dem Mitomycin-Zusatz $3 \mathrm{Stdn}$. verstreichen, so steigt die DNS-Synthese bereits auf etwa $40 \%$ des Kontrollwertes der DNS-Synthese in unbehandelten, infizierten Kulturen. Wird erst nach 6 Stdn. nach der Infektion Mitomycin zugefügt, so kann die DNS-Synthese kaum noch verringert werden; sie beträgt von nun an fast $100 \%$ des Kontrollwertes. Da aber aus solchen Kulturen kein infektiöses Virus hervorgeht, muß entweder die unter Mitomycin-Einfluß neu gebildete Virus-DNS funktionsunfähig oder aber der Komplettierungs-Mechanismus der Virusteilchen gestört sein. Ben-Porat, Reissig und Kaplan ${ }^{9}$ konnten mit Mitomycin die Produktion an infektiösem Pseudorabies-Virus fast gänzlich unterdrücken, fan-

9 T. Ben-Porat, M. Reissig u. A. S. Kaplan, Nature [London] 190, 33 [1961]. 
den aber, daß die Anzahl der physikalischen Teilchen fast genau derjenigen entsprach, die sie in Mitomycin-unbehandelten, infizierten Kontrollkulturen vorfanden.

Alle Mitomycin-Gaben, die später als 12 Stdn. p.i. einsetzen, können nur noch eine weitergehende VirusSynthese verhindern. Die sich einstellende Virusausbeute entspricht von nun an stets genau dem Titer in Kontrollkulturen zur gleichen Zeit (vgl. Abb. 1). Daraus ergibt sich, daß die Komplettierung der HsV-Partikel etwa 12 Stdn. p.i. beginnt und daß einmal fertiggestelltes Virus durch Mitomycin nicht mehr in seinen infektiösen Eigenschaften beeinträchtigt werden kann.

In gewisser Hinsicht unterscheiden sich die am E. coli-System nach Phagen-Infektion und Mitomycin-Behandlung gewonnenen Befunde ${ }^{10,11}$ von unseren Ergebnissen. Hier wird durch den infizierenden $\mathrm{T} 2 \mathrm{r}$-Phagen sofort und auch bei vollständig blockierter Bakterien-DNS-Synthese ein eigener und autonomer DNS-Syntheseweg aufgebaut. Zu keinem

10 M. Sekiguchi u. Y. TAKagi, Biochim. biophysica Acta [Amsterdam] 41, 434 [1960].
Zeitpunkt der Infektion ist eine funktionsfähige Bakterien-DNS Voraussetzung für die Vermehrung des Phagen. Aber auch hier sind die unter hohen Mitomycin-Dosen produzierten Phagen nicht infektiös.

Aus unseren Ergebnissen schließen wir, daß während der ersten Stdn. nach der Infektion neue Enzymsysteme der Virus-DNS-Synthese aufgebaut werden. Erst 6 Stdn. nach der Infektion setzt eine neue Virus-DNS-Synthese ein. Sie ist dann unabhängig von einer replikationsfähigen Zell-DNS und kann durch Mitomycin nur noch so beinflußt werden, daß sie nicht mehr zur Synthese infektiöser Viruspartikel beitragen kann. Die Ursache dafür könnte eine teilweise Degradation der Virus-DNS sein. Zur Zeit versuchen wir, diese Frage durch andere Experimente zu klären.

Die Arbeit wurde mit Unterstützung der Deut schen Forschungsgemeinschaft durchgeführt. Frau U. Selter und Fräulein M. Pernfuss danken wir für ihre Mitarbeit.

11 M. Sekiguchi u. Y. Takagi, Virology 10, 160 [1960]. 